\title{
External quality assurance in histopathology: Experience of the east of Scotland scheme
}

\author{
H S Klys, A M Lessells
}

\begin{abstract}
Aims: To examine the overall organisation of a voluntary external quality assurance scheme in histopathology, selection of case material and marking systems; and to assess the scheme in relation to contemporary histopathological practice.
\end{abstract}

Methods: Case material was derived from all participating departments and a positive marking system was used throughout the scheme.

Results: The mean response rate by those agreeing to participate was $66 \%$ and remains stable. The mean score of each set $(n=8)$ was high $(63-80 \%)$ but scores about the mean varied widely in each set. The positive marking system was satisfactory and provided information equivalent to negative and weighted marking systems. The use of local coordinators providing the link between the central organiser and individuals has spread the administrative load and facilitated the introduction of small biopsy specimens. The selection of suitable case material has proved difficult at times.

Conclusions: The scheme has an important role in histopathology quality assurance. It has been operative for four years and is widely accepted in the east of Scotland; it can be adapted to suit local circumstances.

Medical audit embraces professional, ethical, and financial standards in every stage of patient investigation and management. Quality assurance in histopathology falls within this broad remit. The need for quality assurance schemes is enshrined in the NHS review Working for patients ${ }^{12}$ and is an important element of proposals for laboratory accreditation both in Great Britain and abroad. ${ }^{34}$ Medical audit is actively supported by the medical profession ${ }^{5-7}$ and the principles behind it are both straightforward and widely understood. ${ }^{8}$

Quality assurance schemes may be conveniently divided into two groups: internal quality assurance schemes (IQAS); and external quality assurance schemes (EQAS). IQAS tend to concentrate on technical and clerical aspects of laboratory work in histopathology and are usually organised at a local level. ${ }^{10}$ EQAS are often orientated more to the interpretative aspects of histopathology and are organised on a regional or national basis. ${ }^{11}$ These two broad groups are not mutually exclusive and ideally they should be complementary.

National external quality assurance schemes (NEQAS) have been operating for many years in the fields of clinical chemistry, haematology, and microbiology. ${ }^{12}$ A NEQAS for immunohistochemistry has been in place for some time. ${ }^{13}$ Many histopathologists have felt that the personal and subjective nature of histopathological diagnosis precludes it from external quality assessment, but high standards of diagnostic accuracy depend on the uniform application of recognised criteria. These standards are fundamental to histopathological practice and outweigh any other considerations in the provision of a diagnostic service to clinical staff.

It has become increasingly apparent that the "uniqueness" of histopathological practice cannot be maintained and it must be subjected to audit just like other disciplines. At the same time it is recognised that this is a complex process. ${ }^{14}$

In recognition of the need for EQAS in histopathology the EQA forum was established under the auspices of the Department of Health, and the Scottish Home and Health Department, together with representatives of the Royal College of Pathologists and the Association of Clinical Pathologists. In 1985 the forum produced a protocol ${ }^{15}$ for the introduction of a voluntary EQAS on a regional or supraregional basis. The stated objectives of the scheme are to improve diagnostic accuracy, to reduce health care costs, and to provide a basis for the continuing education of histopathologists in laboratory investigation, organisation, and management.

In 1986 one of the authors (AML) agreed to organise a scheme in the East of Scotland, largely based on the EQA forum model. At the time of writing the scheme has been in operation for four years with completion of eight slide set circulations.

\section{Design of the scheme}

Three university departments (six to 14 consultants or senior lecturers in each) and four NHS departments (two to four consultants in each) participate in the scheme.

From the outset a local coordinator was appointed in each of the participating

\section{Table 1 Role of local coordinator}

1 Copying and distribution of correspondence and answersheets

Distribution of slide sets

Supervise submission of answersheets by the closing date 4 Distribution of code letters 
Table 2 Uptake of scheme and overall scores

\begin{tabular}{lllllllll}
\hline Set & 1 & 2 & 3 & 4 & 5 & 6 & 7 & 8 \\
\hline No. of replies & 21 & 16 & 17 & 16 & 20 & 24 & 20 & 23 \\
Percentage of participants replying 78 & 53 & 57 & 62 & 67 & 75 & 63 & 72 \\
Range of scores (\%) & $18-96$ & $43-89$ & $25-96$ & $23-100$ & $38-96$ & $46-90$ & $36-100$ & $50-100$ \\
Mean score (\%) & 65 & 63 & 64 & 80 & 74 & 69 & 78 & 75 \\
\hline
\end{tabular}

laboratories. The organiser (AML) communicates with the local coordinator rather than individual pathologists. The local coordinator is responsible for photocopying and distribution of letters and answersheets, distribution of slide sets, and supervision of the return of answers by the closing date (table 1). This delegation of administrative responsibility has proved remarkably efficient and has a central role in our scheme.

All cases used throughout the scheme are contributed by participating laboratories. Attempts are made to include cases from each participating laboratory in each circulation. The cases submitted are screened for technical quality and suitability by the organiser, but none has been rejected thus far on the basis of unsuitability.

One slide set is distributed to each NHS department and three or four to each university department. A total of 15 sections are required from each case. This system has facilitated the introduction of small biopsy specimens to the scheme.

Each slide set is composed of 12 slides ( 14 on one occasion). Diagnosis is based on a single haematoxylin and eosin stained slide in each case and neither histochemical nor immunohistochemical stains are given. The answer sheet provides relevant clinical details and a brief macroscropic description where appropriate. It is recommended that each slide set is completed in two sittings of one hour or less and that undue effort is not expended. Access to textbooks and journals is not specifically prohibited.

In practice it has only been possible to have two slide circulations a year in February and September. These dates are designed to avoid the university summer term. A closing date is given at the start of each circulation usually five to six weeks from the time of circulation, but the scheme has always overrun this date.

Anonymity is an essential component of any EQA scheme. In our scheme each participant is assigned a code letter. The code letters are chosen at random and are not known to the organiser. The codes are distributed via the local coordinator in blank envelopes. Answersheets are returned with the code letter as a means of identification. It is strongly recommended that answers are typed to maintain

Table 3 Distribution of cases by anatomic system

\begin{tabular}{lr}
\hline System & $\%$ \\
\hline Skin & 16 \\
Soft tissue & 13 \\
Gastrointestinal & 13 \\
Female reproductive & 13 \\
Lymphoreticular & 12 \\
Respiratory & 8 \\
Male reproductive & 7 \\
Breast & 7 \\
Other & 10 \\
\hline
\end{tabular}

anonymity. In practice several participants ignore this and send in signed replies.

At the start of the scheme a summary of the submitted diagnoses was distributed together with the preferred ("correct") diagnoses. After four circulations a formal marking system was introduced with retrospective marking of the earlier circulations. Each answer received a mark of $0,0.5$, or 1 . Negative marking was not used. All marking and collation of results was carried out by the organiser. The distribution of scores and marks for each question are sent to the participants, allowing them to calculate their own personal total score. A breakdown of performance on an individual basis was not given.

\section{Results}

A summary of the participants' scores and the level of participation is shown in table 2 . The actual number of replies to each circulation is given in the first rank and this number, expressed as a percentage of the total of participants, is seen in the second rank. The mean reply rate was $66 \%$ of those expressing an interest in the scheme.

The mean score of each set was high (63$80 \%$ ). In general there was a wide range of scores in each circulation. The scores in the eight sets correspond roughly to a normal or Gaussian distribution. This property aids statistical analysis, in particular the comparison of performance among different groups. It is also an indication that the selected cases are likely to be of appropriate difficulty.

\section{SELECTION OF CASES}

The success of the scheme is hinged on the cases submitted for examination. They must reflect the normal departmental workload and, while being acceptable to the participants, must attain an appropriate level of difficulty. Any individual case or set of cases will fulfil these criteria to a greater or lesser degree.

The anatomical site of origin of all our cases is given in table 3 . The distribution reflects the working situation, but possibly with a bias to areas where diagnostic problems more frequently arise.

Table 4 illustrates the range of material and preferred diagnoses for slide set 6 , which we regard as representative of our scheme. Cases 1 , $2,4,7,8$ and 9 are, in our opinion, the most discriminatory. Cases 3 and 12 were answered correctly by all respondents and are therefore non-discriminatory. As in these cases their suitability only becomes obvious after marking and analysis of the results. Case 5 we would regard as too difficult for discriminatory purposes, but it is of educational value. The EQA forum recommended that in cases where genuine differences of opinion occurred the help of a recognised expert should be sought. In cases 
Figure 1 Comparison of scoring systems.
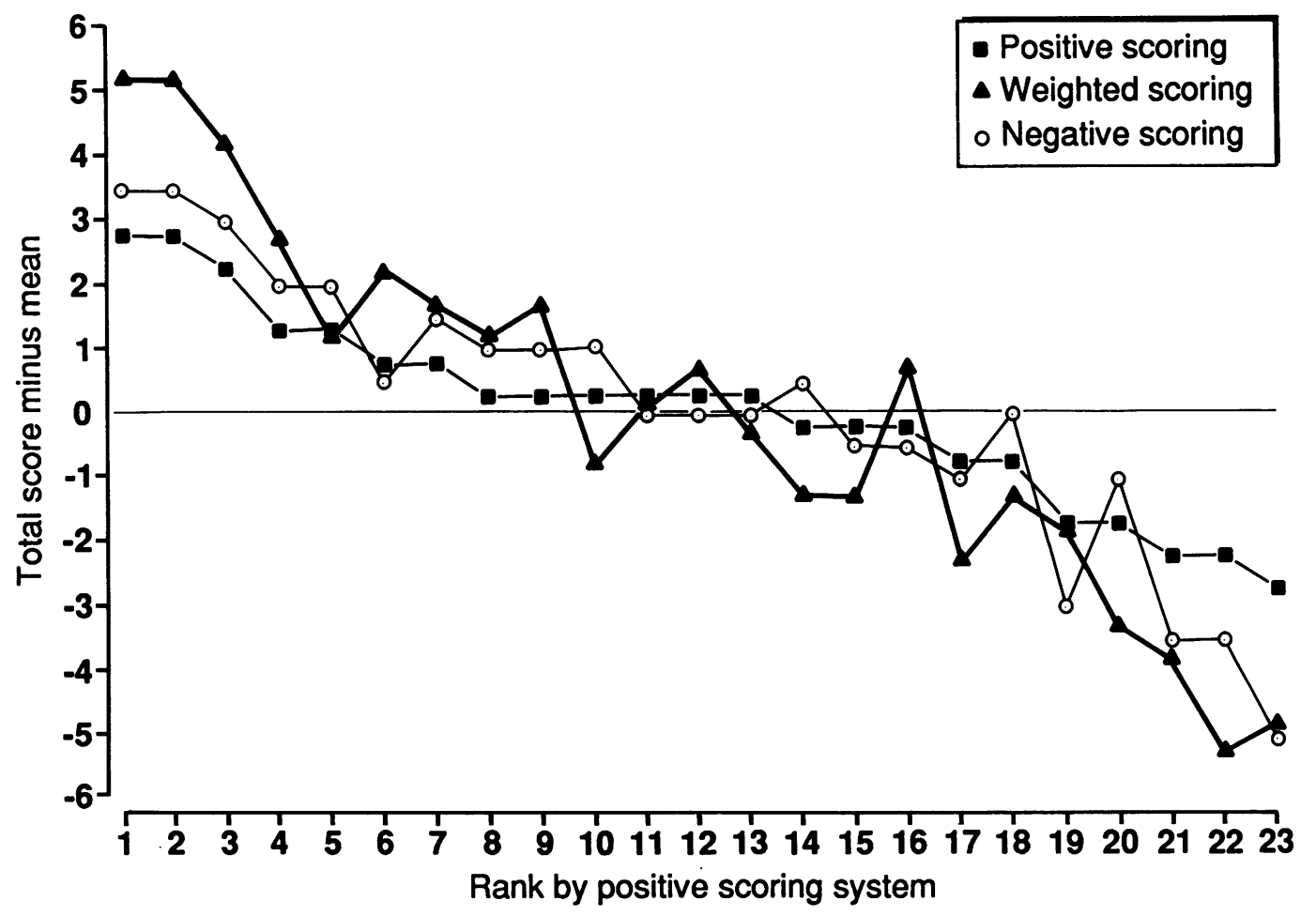

Table 4 Case details from $E Q A$ set 6

\begin{tabular}{rrlllr}
\hline $\begin{array}{l}\text { Case } \\
\text { No. }\end{array}$ & Age & Sex & Specimen & Preferred diagnosis & $\begin{array}{c}\text { Correctly } \\
\text { answered (\%) }\end{array}$ \\
\hline 1 & 31 & M & Open biopsy of lung & Histiocytosis X & 75 \\
2 & 30 & M & Mass at ankle & Tenosynovial giant cell tumour & 63 \\
3 & 28 & F & Cervical lymph nodes & Metastatic papillary carcinoma & 100 \\
4 & 85 & M & Gastric tumour & Epithelioid leiomyoma & 75 \\
5 & 9 & F & Mass on forearm & Angiomatoid MFH & 29 \\
6 & 19 & F & Uterine curettings & Placental site reaction & 38 \\
7 & 62 & F & Open lung biopsy & Bronchocentric granulomatosis & 58 \\
8 & 38 & F & Cervical biopsy & Granulocytic sarcoma & 67 \\
9 & 25 & M & Mass from elbow & Cat scratch disease & 71 \\
10 & 57 & M & Laryngeal biopsy & Verrucous carcinoma & 25 \\
11 & 60 & M & Nasal polyps & Well differentiated adenocarcinoma & 13 \\
12 & 4 & F & Bone biopsy & Eosinophilic granuloma & 100 \\
\hline
\end{tabular}

10 and 11 an outside opinion was sought before the cases were submitted. In case 6 most respondents gave a diagnosis of placental site trophoblastic tumour (PSTT). This case was subsequently referred to a gynaecological reference pathologist who agreed with the original diagnosis of placental site reaction. Unfortunately, the patient later developed pulmonary metastases of PSTT, proving that the opinion of the expert is not guaranteed to be correct. Clinical followup data may be required as the ultimate arbiter in some cases.

In case 8 the original pathological diagnosis on the evidence of the cervical biopsy specimen was that of carcinoma. At hysterectomy this diagnosis was rejected and the lesion was

Table 5 Variety of terminology in one case

Adenocarcinoma? mucoepidermoid with spindle cell changes Carcinoma of mixed differentiation

Carcinosarcoma

Bronchioalveolar carcinoma

Mucoepidermoid carcinoma

Spindle cell carcinoma

Spindle cell squamous carcinoma

High grade carcinoma with spindle cell areas

Adenocarcinoma

Adenocarcinoma with spindle cell elements

Metaplastic carcinoma

Poorly differentiated adenocarcinoma/large cell carcinoma Carcinoma-large cell? bronchioloalveolar

Squamous carcinoma with spindle cell and clear cell elements Large cell carcinoma with spindle cell and adenocarcinoma components classified as lymphoma. The EQA participants showed that most favoured a diagnosis of lymphoma with two suggesting leukaemia. Either diagnosis was accepted. It was only several months later that the patient developed acute myeloid leukaemia and the true nature of the cervical lesion became apparent.

\section{NOMENCLATURE}

Throughout the scheme a lack of uniformity in terminology has presented problems which may be reflected in working practice. An example is given in table 5 . Eight of the 23 respondents gave the diagnosis of carcinosarcoma leaving 14 different diagnoses from 15 pathologists.

\section{SCORING SYSTEMS}

A comparison of the positive scoring system used throughout the scheme with a punitive negative scoring system and a weighted marking system is shown (figure) using data from set 8. The rank under a positive scoring system is plotted on the $x$-axis. The actual score minus the mean $(s-x)$ is plotted on the $y$-axis. Changes of rank order as a result of using a different scoring system occur where any plotted lines cross. It can be seen that the different systems do not significantly alter outcome, but exaggerate differences at the upper and lower ends of the scale. 


\section{Discussion}

The introduction of a voluntary EQA scheme in the east of Scotland has been greeted with enthusiasm by most consultant histopathologists in the area. Over the four year period interest has been maintained and the participation level has remained steady. Support for the scheme has been stronger in the four nonuniversity departments.

The scheme has been set up and developed with modest administrative effort and minimal cost, with the EQA forum protocol as a starting point. The chief deviation from the protocol has been the introduction of local coordinators and consequent distribution of the administrative load.

To what extent does the scheme achieve the stated aims of the EQA forum and what is its role in audit and maintaining standards? Our scheme has adequately fulfilled the role of continuing education and to an extent this overlaps with the operation and aims of a slide club. In particular, the inclusion of unusual and recently described entities has been important, but the use of single haematoxylin and eosin slides is an obvious limitation. The scheme is only of indirect relevance to laboratory organisation and management. The contribution of an EQA scheme to reducing health care costs, although laudable, cannot be reliably assessed.

The aim of maintaining and improving standards of diagnostic accuracy are the most difficult to address and form the focus of the controversy surrounding EQAS in histopathology. ${ }^{9}$ Quality assurance is a measure of outcome. ${ }^{16}$ The pathologist's report is regarded as the final outcome of a histopathological investigation, although it is an intermediate event in patient management. The EQAS can be regarded as a form of proficiency testing and is therefore an indirect measure of outcome. We have to assume that proficiency in slide diagnosis within the scheme is reflected in consultant performance in the working situation. This extrapolation of results forms a possible weak link yet EQA schemes based on proficiency testing are widely accepted in other fields.

The main criteria of any form of testing are reliability, validity, appropriate difficulty and discriminatory power. These are open to statistical analysis in some circumstances for example, large scale university examinations, but the small numbers and subjectivity preclude this approach in the EQA setting. Given these constraints it is evident that the selection of case material and the marking of answers is critical both to the acceptability and usefulness of the scheme. We have found that the policy of using material contributed by participants greatly enhances acceptability. Prompt feedback with case analysis and distribution of scores is of great importance. Despite careful case selection we have found that difficulties in areas such as nomenclature still arise and that this may complicate marking. We have encountered difficulties in establishing correct diagnoses in certain rare cases despite using a reference pathologist.

The possible introduction of a compulsory EQAS in histopathology raises several problems, and would necessarily differ from the voluntary scheme described above in a number of areas. Administration would be more complex, both to maintain absolute confidentiality and to avoid collusion between pathologists. This would require formal funding and supervision by a recognised body. Despite the recent emphasis laid on medical audit it is apparent that appropriate funding may be difficult to obtain. ${ }^{10}$ Selection of cases would be more complex due to the requirement to cater for differing degrees of subspecialisation and other commitments.

We believe that the EQAS outlined in this paper has a role in quality assurance and in continuing education, although we accept its limitations. The process of histopathological diagnosis is so fundamental in our specialty that it warrants separate consideration. The EQA forum model provides a suitable basis for this process and we have shown that it can be adapted to suit local circumstances. Ideally, this type of scheme would run alongside a locally organised IQAS which concentrates on the day to day running of a laboratory.

Participation in EQA in some form will probably become a contractual obligation for consultant staff and this is implied in the NHS review. ${ }^{2}$ It is also stated that "professional leadership is essential" and that "the quality of medical work can only be reviewed by a doctor's peers". Participation in a recognised EQAS may be required for accreditation of laboratories and we would regard the above scheme as suitable for this purpose. In what is likely to be an increasingly competitive and possibly commercial future for laboratories it is important that diagnostic pathologists should adopt an urgent and positive attitude towards EQA schemes.

We thank Drs F D Lee and R A Burnett for their advice in the design and setting up of the scheme. We also thank the pathology consultant staff in the east of Scotland for their continuing support.

1 Department of Health. Working for Patients. London: HMSO, 1989.

2 Department of Health. Working Paper 6 on The Health Service. London: HMSO, 1989.

3 Commission on Laboratory Accreditation. Standards for Laboratory Accreditation. Washington, DC: College of American Pathologists, 1988.

4 The College Accreditation Steering Committee. Royal College of Pathologists' United Kingdom pilot study of laboratory accreditation. J Clin Pathol 1990;43:89-91.

5 van't Hoff W. Welcome for medical audit. Br Med $J$ 1989;298:1021-3.

6 Royal College of Physicians. Medical Audit-a first report: what, why and how? London: Royal College of Pathologists, 1989.

7 Royal College of Pathologists. Comments of the Royal College of Pathologists on the White Paper "Working for Patients" and the associated Working Papers for England. Bulletin of the Royal College of Pathologists. 1989;67:2-3.

8 Shaw LD, Costain DW. Guidelines for medical audit: seven principles. Br Med J 1989;299:498-9.

9 Lee FD. External Quality Assessment in Histopathology: an overview. J Clin Pathol 1989;42:1009-11.

10 Ramsay AD. Locally organised medical audit in histopathology. J Clin Pathol 1991;44:353-7.

11 Lee FD, Burnett RA. Quality assurance in histopathology. $J$ Pathol 1987;152:247-51.

12 Ward PG, Lewis SM. Interlaboratory trials: a national proficiency scheme in Britain. Quality control in haematology. London: Academic Press, 1975:37-51.

13 Reynolds GJ. External quality assurance and assessment in immunocytochemistry. Histopathology 1989;15:627-33.

14 Management Advisory Service to the NHS. Review of Pathology Services. Staffing and Management. Cheltenham: $C O I, 1989: 4$.

15 External Quality Assessment Scheme in Histopathology: Bulletin of Royal College of Pathologists 1986;56:3-4.

16 Travers $H$. Quality assurance indicators in anatomic pathology. Arch Pathol Lab Med 1990;114:1149-56. 